\title{
Preparation and Characterization of Poly(vinyl alcohol)-chondroitin Sulphate Hydrogel as Scaffolds for Articular Cartilage Regeneration
}

\author{
Shivani Nanda, ${ }^{1}$ Nikhil Sood, ${ }^{1}$ B. V. K. Reddy, ${ }^{2}$ and Tanmay S. Markandeywar ${ }^{1}$ \\ ${ }^{1}$ Nanomedical Research Centre, ISF College of Pharmacy, VPO-Ghal Kalan, Ferozepur G.T. Road, Moga, Punjab 142001, India \\ ${ }^{2}$ Department of Pharmacology, ISF College of Pharmacy, VPO-Ghal Kalan, Ferozepur G.T. Road, Moga, Punjab 142001, India \\ Correspondence should be addressed to Shivani Nanda; shanu05nanda@gmail.com
}

Received 31 July 2013; Accepted 19 September 2013

Academic Editors: A. Bhattacharyya and S. Dhara

Copyright (C) 2013 Shivani Nanda et al. This is an open access article distributed under the Creative Commons Attribution License, which permits unrestricted use, distribution, and reproduction in any medium, provided the original work is properly cited.

The aim of the study was to develop PVA-CS hydrogel scaffolds using glutaraldehyde as a cross-linking agent by chemical crosslinking method in order to obtain biomimetic scaffolds for articular cartilage regeneration. The introduction of PVA enhances the mechanical and bioadhesive properties to the native tissue while chondroitin sulphate enhances the glycosaminoglycan content of extracellular matrix. The role of hydrogel as cartilage regeneration scaffold was evaluated by swelling study, porosity, rheological behaviour, in vitro degradation, and quantification of released chondroitin sulphate. In vivo results showed that cross-linked hydrogels repaired defects with no sign of inflammation as it was well anchored to tissue in the formation of new articular surface. It may be concluded that the addition of chondroitin sulphate to the PVA polymer develops a novel composite with significant applications in cartilage tissue engineering.

\section{Introduction}

Hydrogels are naturally derived macromolecules having characteristic features like biocompatibility, cell-controlled degradability, and intrinsic cellular interaction which ultimately influence their application in tissue engineering, particularly for growth of new tissues $[1,2]$. Hydrogels are mostly favourable for promoting cell migration, angiogenesis, high water content, and enhanced nutrient diffusion [3]. Hydrogels are prepared from synthetic or natural polymers, which are cross-linked through either covalent or noncovalent bonds [4].

Articular cartilage injuries are major concern in today's clinical practice. Current surgical techniques to repair osteochondral defects are associated with poor cartilage regeneration and tissue formation which results in joint pain and stiffness. The lack of vascularisation of the articular cartilage, however, prevents the development of an inflammatory response which limits the spontaneous repair [5]. This drawback has shifted research interest towards the field of biomaterials and bioengineering, where it is hoped that the cartilage repair can be tackled with the help of tissue engineering. Polymeric scaffolds include biomaterials designing by incorporating the desired guest molecules to promote cell survival and tissue regeneration [6].

The hydrogel scaffolds are used in the engineering of replacement connective tissues which is primarily due to their biochemical similarity to the highly hydrated GAG components of connective tissues. Scaffolds have got attention due to their ability to mimic the mechanical and biological properties of the tissue matrix and thus considered as a special tool for tissue engineering from past two decades [7].

Glycosaminoglycans (GAGs), mainly for chondroitin sulfate (CS), are an important component of the extracellular matrix (ECM) of cartilage and participate in the regeneration of cartilage defects. CS is the most abundant GAG and varies from 20 to $80 \%$ of total GAGs [8]. Incorporation of chondroitin sulfate in hydrogel scaffold may promote the secretion of proteoglycan which provides compressive strength, while type II collagen provides tensile strength 
which influences the bioactivity of the seeded chondrocytes [9] and performs important biological functions, such as regulating cell adhesion and motility and mediating cell proliferation and differentiation, which is presented in high amounts in the ECM during embryonic cartilage development, and also facilitates the integration of engineered cartilage $[10,11]$.

Polyvinyl alcohol is polymer of great interest in cartilage regeneration because of its many desirable characteristics specifically good biocompatibility and suitable mechanical strength [12]. PVA is capable of simulating natural tissue and can be accepted into the body because of its high degree of swelling in water and biological fluids and its rubbery and elastic nature. PVA must be cross-linked in order to remain insoluble in solution, the crosslink may be accomplished by chemical agents (glutaraldehyde, acetaldehyde, and formaldehyde) by the use of electron beam orirradiation, under alkaline conditions and by physical methods such as repeated freeze-thawing cycles [13].

In this study, PVA-CS hybrid hydrogel scaffold that partly mimicked the composition and structure of cartilage extracellular matrix was synthesized by chemical-cross linking method. Chondroitin sulfate and PVA were crosslinked by glutaraldehyde to achieve the main objective of the study related to the biodegradability of composite scaffold at a controllable rate that approximates the rate of cartilage regeneration $[14,15]$.

\section{Materials and Method}

2.1. Materials. The biomaterial chondroitin sulphate was obtained as a gift sample from Ambe Extracts, New Delhi, polyvinyl alcohol (PVA), glutaraldehyde, and acetic acid was obtained from $\mathrm{CDH}$ Analytical Reagents, New Delhi and sulphuric acid was obtained from HiMedia Laboratories Pvt. Ltd., Mumbai.

\subsection{Preparation of PVA-CS Hydrogel Scaffold Formulations.} PVA-CS hydrogel scaffold formulation was fabricated using chemical cross-linking method Table 1. In this method, PVA was dissolved in hot water at $80-90^{\circ} \mathrm{C}$ for 1 hour and then allowed to cool at room temperature. Then chondroitin sulphate was added to PVA solution further allowing mixing with $30 \mathrm{~mL}$ of $10 \% \mathrm{v} / \mathrm{v}$ of aqueous solution of acetic acid and $10 \mathrm{~mL}$ of $1 \% \mathrm{v} / \mathrm{v}$ aqueous solution of sulphuric acid. Then aqueous solution of glutaraldehyde (\% v/v) was added dropwise as cross-linking agent. The reactants were stirred thoroughly, and the mixtures were poured into petri dishes for 48 hours at room temperature to obtain polymeric hydrogel scaffolds and petri dishes were covered to prevent evaporation [16].

2.3. Rheological Studies. Rheological properties of hydrogel scaffolds were monitored using rotating Rheometer (Brookfield Engineering Laboratories, USA, R/S plus) equipped with temperature controller and spindle (C 75-1). Viscosity of samples was measured at different shear rate. The shear rate was increased from $0-5 \mathrm{~s}^{-1}$ in $1 \mathrm{~min}$. The viscosity was
TABLE 1: Preparation scheme of PVA-CS hydrogel scaffold formulations.

\begin{tabular}{lccc}
\hline $\begin{array}{l}\text { Formulation } \\
\text { code }\end{array}$ & PVA w/v (\%) & GA v/v (\%) & $\begin{array}{c}\text { Chondroitin } \\
\text { sulphate (mg) }\end{array}$ \\
\hline F1A & 5 & 0.5 & - \\
F2A & 5 & 1.0 & - \\
F3A & 5 & 1.5 & - \\
F4A & 5 & 2.0 & - \\
F5B & 10 & 0.5 & - \\
F6B & 10 & 1.0 & - \\
F7B & 10 & 1.5 & - \\
F8B & 10 & 2.0 & - \\
F9C & 15 & 0.5 & - \\
F10C & 15 & 1.0 & 100 \\
F11C & 15 & 1.5 & 100 \\
F12C & 15 & 2.0 & 100 \\
F13D & 20 & 0.5 & 100 \\
F14D & 20 & 1.0 & - \\
F15D & 20 & 1.5 & - \\
F16D & 20 & 2.0 & - \\
\hline
\end{tabular}

determined from the flow curve obtained at different values of shear rate. All measurements were made in triplicate [17].

2.4. Equilibrium Swelling Ratio of Hydrogel Scaffolds. The gravimetric method was used for equilibrium swelling ratio (ESR) determination of hydrogel samples. Briefly, hydrogels were dried in vacuum oven for $24 \mathrm{hrs}$ at $45^{\circ} \mathrm{C}$. The dried hydrogel disc of (Approx. $10 \mathrm{mg}$ ) was immersed in distilled water, PBS (pH 1.2, 6.5 and 7.4) at $37 \pm 0.2^{\circ} \mathrm{C}$ in an incubator. Swollen discs were lifted at preset time interval of 1, 2, 3, 4, 5, 6, 12 and 24 hours, till equilibrium was achieved, blotted on wet filter paper for removal of excess of water from the surface and weighed on analytical digital balance $(n=3)$. The ESR was calculated using following formula (see [18]):

$$
\mathrm{ESR}=\frac{W_{t}-W_{i}}{W_{i}} \times 100,
$$

where ESR is equilibrium swelling ratio, $W_{i}$ is initial weight of the dry hydrogel disc, and $W_{t}$ is weight of the swollen polymer at time $t$.

2.5. Porosity Measurements of Hydrogel Scaffolds. For porosity determination the solvent replacement method was used. Dried hydrogels were immersed overnight in absolute ethanol and weighed after excess ethanol on the surface was blotted. The porosity was calculated from the following equation (see [19]):

$$
\text { Porosity }=\frac{\left(M_{2}-M_{1}\right)}{\rho V} \times 100,
$$

where $M_{1}$ is mass of the hydrogel before immersion in absolute ethanol, $M_{2}$ is mass of the hydrogel after immersion 
in absolute ethanol $\rho$ is density of absolute ethanol, and $V$ is volume of the hydrogel.

2.6. Water Absorption Studies of Hydrogel Scaffolds. The \% water absorbed by the scaffolds was studied by soaking them overnight in distilled water at room temperature. The $\%$ water absorbed was calculated from the following equation (see [20]):

$$
\% \text { Water absorption }=\frac{S-W}{W} \times 100,
$$

where $W$ is Initial weight of scaffold formulations (mg), and $S$ is Weight of scaffold formulations (mg) after soaking the water until saturation (after $24 \mathrm{hr}$ ).

2.7. Apparent Density of Hydrogel Scaffolds. The apparent density of hydrogel scaffolds was studied by soaking them overnight in distilled water at room temperature. The apparent density of the scaffolds was calculated from the following equation (see [21]):

$$
\rho=\frac{4 m}{\pi d^{2} h}
$$

where $\rho$ is Apparent density of porous material $\left(\mathrm{g} / \mathrm{cm}^{3}\right), m$ is Mass of scaffold formulations after saturation with water (gm), $d$ is Diameter of scaffold formulations after saturation with water $(\mathrm{cm})$, and $h$ is Height of scaffold formulations after saturation with water $(\mathrm{cm})$.

2.8. Quantitative Estimation of Released Chondroitin Sulfate by Hydrogel Scaffolds. The release studies were performed on hydrogel scaffolds dried after preparation. Drying before study is carried out to obtain constant weight which avoids interference with swelling of hydrogels and also with the release of CS from its pores. Briefly, the dried hydrogels were incubated in water at $37^{\circ} \mathrm{C}$. The amount of CS released from PVA-CS hydrogel scaffolds was quantified by taking $10 \mu \mathrm{L}$ of the swelling medium added with optimised concentration of $\mathrm{MB}$ dye solution. The released amount of chondroitin sulphate was analyzed at $\lambda_{\max } 664 \mathrm{~nm}$ with reference to water as a blank using UV-visible spectrophotometer [22].

2.9. In Vitro Weight Loss Studies. In vitro weight loss profile of the hydrogels was studied by measuring the change in weight as a function of time. Dry hydrogel discs (approximately

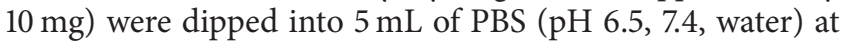
$37^{\circ} \mathrm{C}$. The media were changed every day. At predetermined time interval, samples were withdrawn from the media washed with distilled water and then dried in vacuum oven at $45^{\circ} \mathrm{C}$ till constant weight was obtained. The percentage (\%) weight loss was calculated using the following formula (see [23]):

$$
\% \text { Weight loss }=\frac{\mathrm{Wt}_{(i)}-\mathrm{Wt}_{(t)}}{\mathrm{Wt}_{(i)}} \times 100,
$$

where $\mathrm{Wt}_{(i)}$ is the initial weight of the disc at time zero and $\mathrm{Wt}_{(t)}$ is the weight of the disc at time $t$.
2.10. Morphological Studies (SEM) of Hydrogel Scaffolds. The scaffold formulations were characterized for their internal morphology like structure, shape, and surface morphology using scanning electron microscopy (SEM) (Joel, Japan, EVO-50). Fully swollen hydrogels were lyophilizeds and then they were mounted on to the base platform for gold coating by vapour deposition technique using vacuum sputter coater. Average diameters of all type of scaffold formulations studied were calculated based on the sizes measured from the SEM images using Image J software [24].

2.11. Hardness (Texture Analyzer) of Hydrogel Scaffolds. The hardness of hydrogel was measured by using texture analyzer (Brookfield Engineering Laboratories, USA, CT3). The sample were placed on the platform and probe was travelled to the surface of swollen hydrogel with speed of $0.5 \mathrm{~mm} / \mathrm{s}$; the probe kept moving until reaching the base and the hardness, deformation at hardness, and adhesive force were calculated [25].

2.12. In Vivo Studies. Wistar rats were anesthetized by intramuscular injection of $35 \mathrm{mg} / \mathrm{kg}$ ketamine and knee was exposed and accessed by a medial parapatellar incision, and a cylindrical hole $2 \mathrm{~mm}$ deep $\times 2.5 \mathrm{~mm}$ in diameter was made in the intercondylar region of the femur. The orifice was washed with saline solution and a sample of hydrogel scaffold of the same size as the orifice was implanted in the left knee while the control orifice in the right knee remained empty. The animals were divided into three groups of six animals per group and were observed. Defect repair efficacy of developed formulations will be evaluated for histological analysis after the 14 days of implantation. The specimens were fixed in $10 \%$ paraformaldehyde solution for 48 hours, decalcified with $5.0 \%(\mathrm{v} / \mathrm{v})$ nitric acid, washed and dehydrated with a gradual series of ethanol and xylene solutions. After this, they were embedded in wax blocks and sagittal sections including the adjacent repair tissue which were cut using a rotary microtome and stained with Masson's trichrome reagent under magnification power of $200 \mu \mathrm{m}$. The stained sections were observed and photomicrographed using a light microscope equipped with an automatic exposure photomicrograph system [26].

\section{Results and Discussions}

3.1. Rheological Behaviour of PVA Hydrogel Scaffold Formulations. The viscosity of hydrogel scaffold formulations at different shear rates $\left(0-5 \mathrm{~s}^{-1}\right)$ was obtained as shown graphically in Figure 1. It was observed that all the formulations showed pseudoplastic flow which means that increase in the shear rate will decrease the viscosity and optimum steep curve of graph shows better pseudoplastic behaviour as obtained for F11C formulation (red marked). This might be due to presence of free hydroxyl groups which are higher enough to hold the cross-linking agent concentration to show saturated viscosity. Rheological behaviour of hydrogels depends on the concentration of the polymer and cross-linking agent which is required to be optimum for better flow properties. 


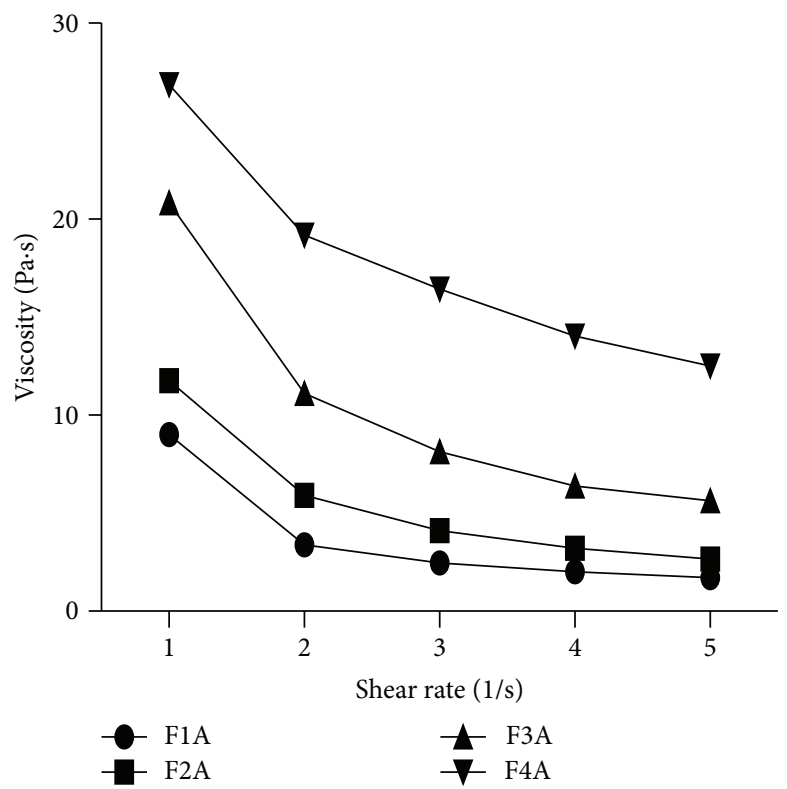

(a)

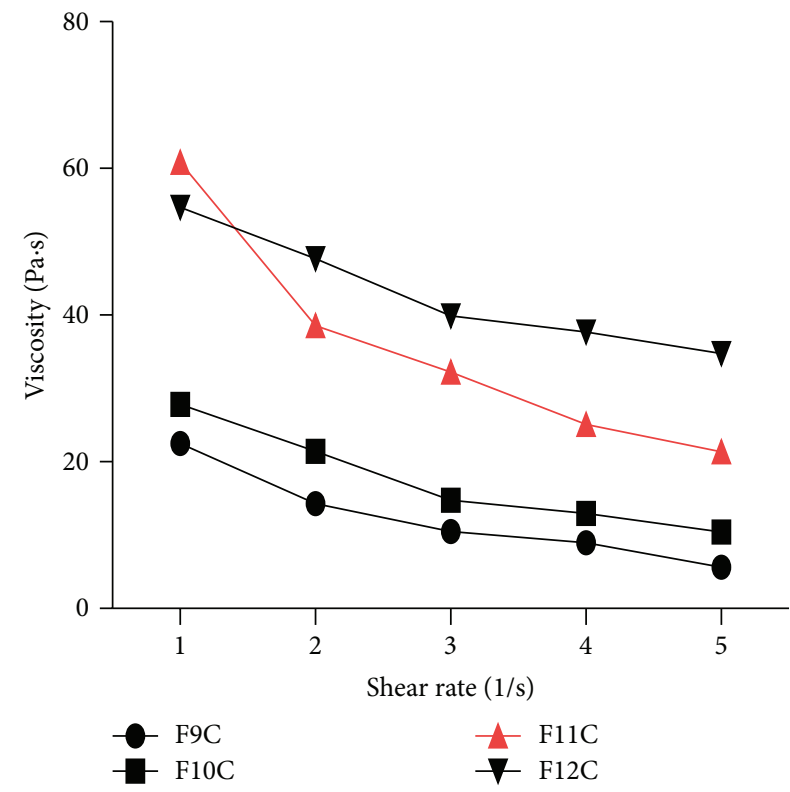

(c)

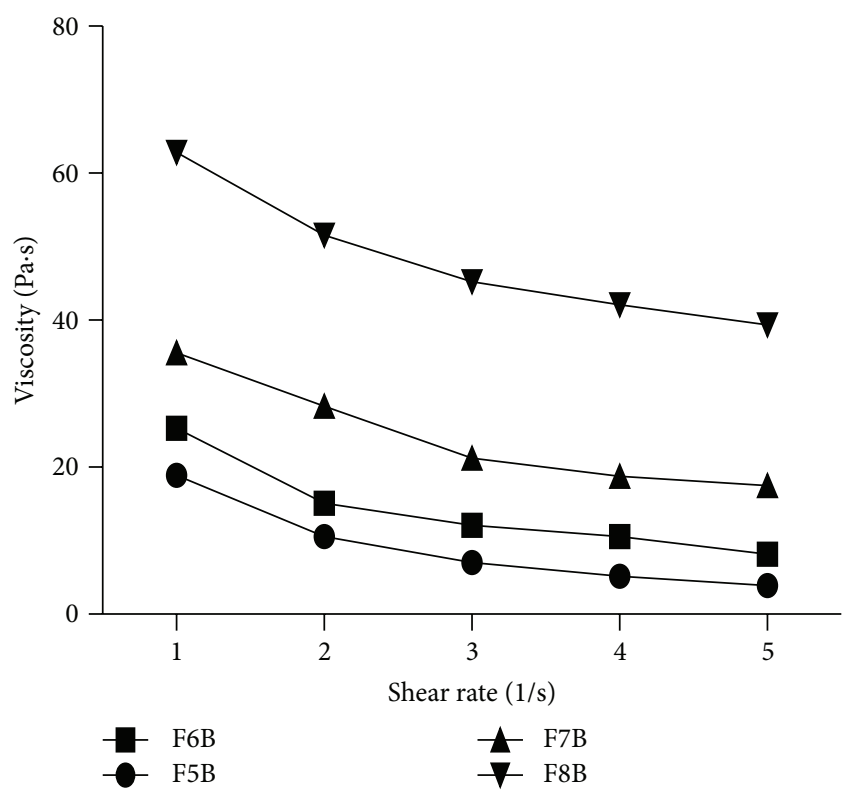

(b)

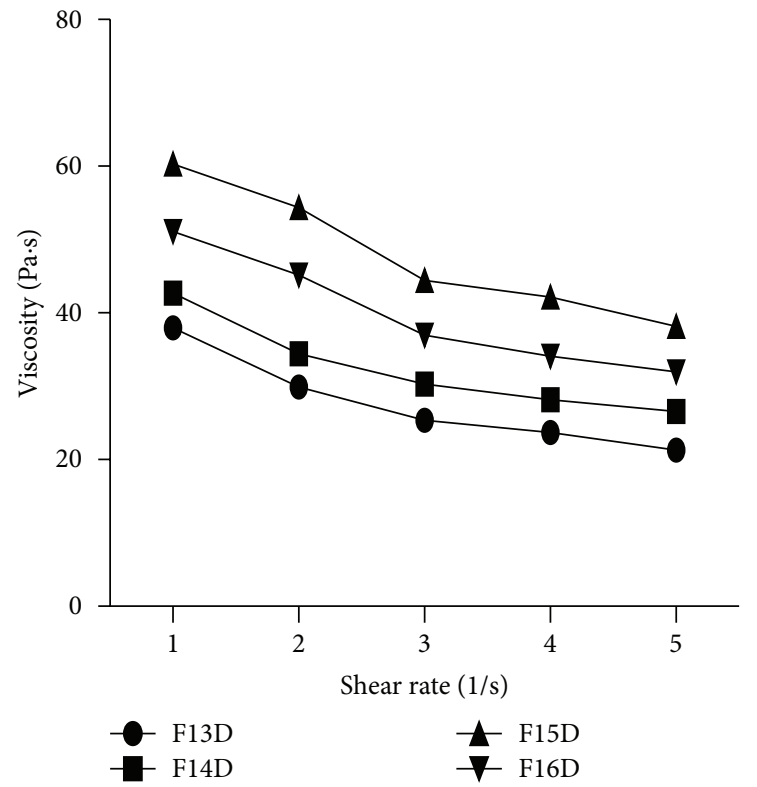

(d)

FIGURE 1: Rheological behaviour of PVA hydrogel scaffold formulations at different shear rates $\left(1-5 \mathrm{~s}^{-1}\right)$.

3.2. Swelling Behaviour of PVA Hydrogel Scaffold Formulations. The \% swelling of hydrogel scaffold formulations in water, PBS buffers of $\mathrm{pH} 1.2,6.5$ and 7.4 were obtained as shown in Figure 2. It was observed that there was no significant difference between the swelling percentages of hydrogel scaffolds in different $\mathrm{pH}(1.2,6.5$, and 7.4) which is due to neutral behaviour of the PVA which does not respond to any environmental stimuli. Penetration of solvent leads to swelling of the hydrogel which is due to diffusion of solventmolecules through the polymer matrix and local relaxation of polymer segments. For rapid relaxation rates, penetration speed is limited by diffusion process and the normal Fickian transport is observed. In this type of swelling mechanism, diffusion of water molecules inside the polymer is a rate-limiting step.

3.3. Porosity Measurements of Hydrogel Scaffolds. The \% porosity of scaffold formulations was obtained as shown in Figure 3. It was observed that porosity depends on the volume of the pores present in the hydrogel scaffolds. The porosity of hydrogels decreased with increase in concentration of glutaraldehyde. This is due to the incorporation of the higher cross-link density within polymer structure leading to decrease in the occupied volume while in case of higher 


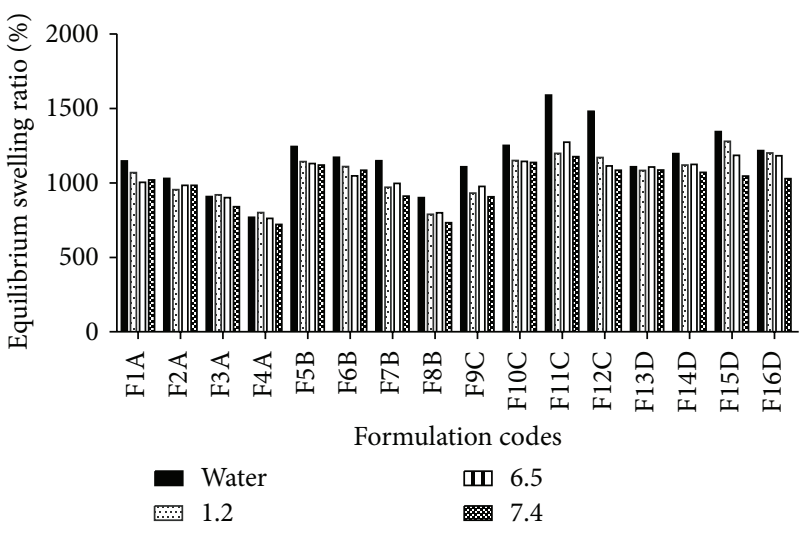

FIgURE 2: Comparative swelling behaviour of PVA hydrogel scaffolds in $\mathrm{pH} 1.2,6.5,7.4$ and water.

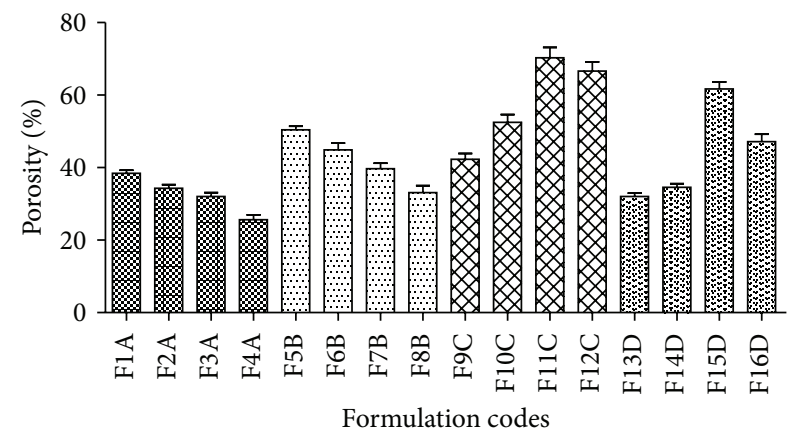

Figure 3: Porosity of PVA hydrogel scaffold formulations.

concentration of PVA the lower cross-link density within the polymer structure lead to increases in the \% porosity. Here in F9C and F10 formulation, the minimum concentration of cross-linking agent was not sufficient to bind 15\% PVA concentration; therefore, it exhibits lower porosity. In F11C formulation maximum porosity was found having required cross-linking density strong enough to keep optimized volume of pores while in F12C due to the incorporation of the higher cross-link density within the polymer structure, it leads to the decrease in the volume of pores in hydrogels.

\subsection{Water Absorption of PVA Hydrogel Scaffold Formulations.}

The $\%$ water absorption of hydrogel scaffold formulations was obtained as shown graphically in Figure 4. It was observed that the water absorption studies depend upon the distribution pattern of pores present within the polymeric structure which further influence the mechanical properties of hydrogel scaffolds. The interconnected pores allowed the polymer to hold more water by capillary force. The percentage water absorption was calculated after the swelling studies in water for $24 \mathrm{hr}$. Increasing the concentration of polymer content up to $10 \%$ and cross-linking agent $0.5 \%$ to $2 \%$ decreases the water absorption, while 15\% PVA content with $1.5 \%$ glutaraldehyde concentration showed higher water absorption percentage because of higher interconnected pore volume.

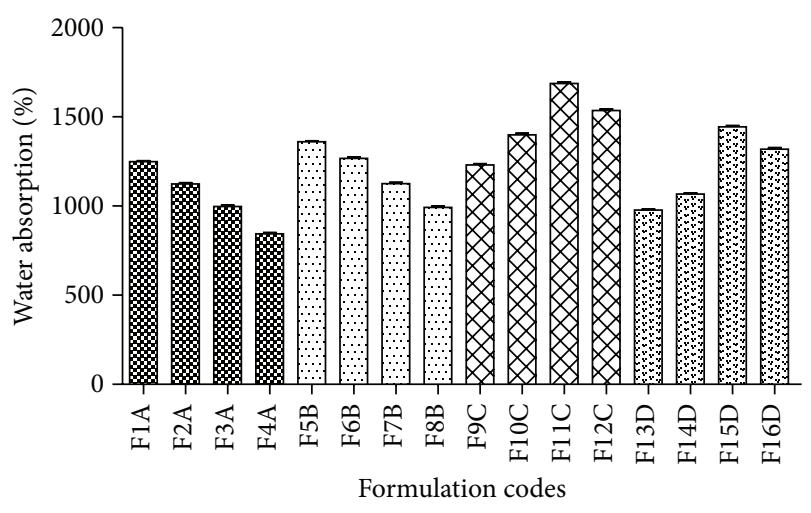

FIGURE 4: Water absorption of PVA hydrogel scaffold formulations.

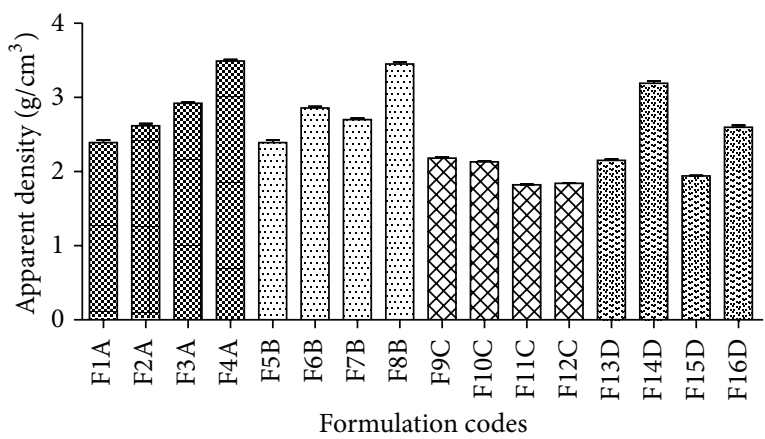

FIGURE 5: Apparent bulk density of PVA hydrogel scaffold formulation.

3.5. Apparent Bulk Density of PVA Hydrogel Scaffold Formulations. The apparent bulk density of scaffold formulations was obtained as shown in Figure 5, and it provides information about pore size and distribution, permeability, and presence of structural faults in polymer structures. It was observed that apparent density of scaffold increase with the increases in concentration of cross-linking agent, while least density was achieved in F11C formulation because of higher concentration of polymer (15\% PVA) and amount of cross-linking agent was not sufficient to crosslink the hydrogels by which it lowers the density while in case of F9C and F10C formulation due to lesser crosslinking concentration, apparent bulk density is higher. The apparent density of a porous scaffold can influence its mechanical strength, permeability, and presence of structural defects.

3.6. Selection of Optimized Formulations. All formulations (F1A-F4A, F5B-F8B, F9C-F12C, and F13D-F16D) were evaluated using parameters like \% swelling index, viscosity $(\mathrm{Pa})$, apparent bulk density $\left(\mathrm{g} / \mathrm{cm}^{3}\right)$, \% water absorption, and $\%$ porosity. F11C formulation was found to be optimized on basis of these parameters as shown in Table 2.

3.7. Quantification of Amount of Released Chondroitin Sulphate from PVA-CS Hydrogel Scaffold Formulations. The \% in vitro cumulative drug release from scaffold formulations 


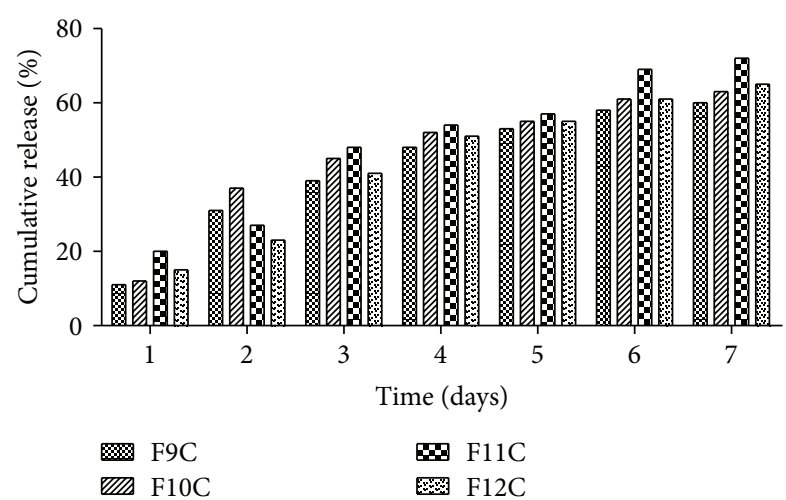

FIGURE 6: In vitro cumulative CS release from PVA-CS hydrogel scaffold formulation.

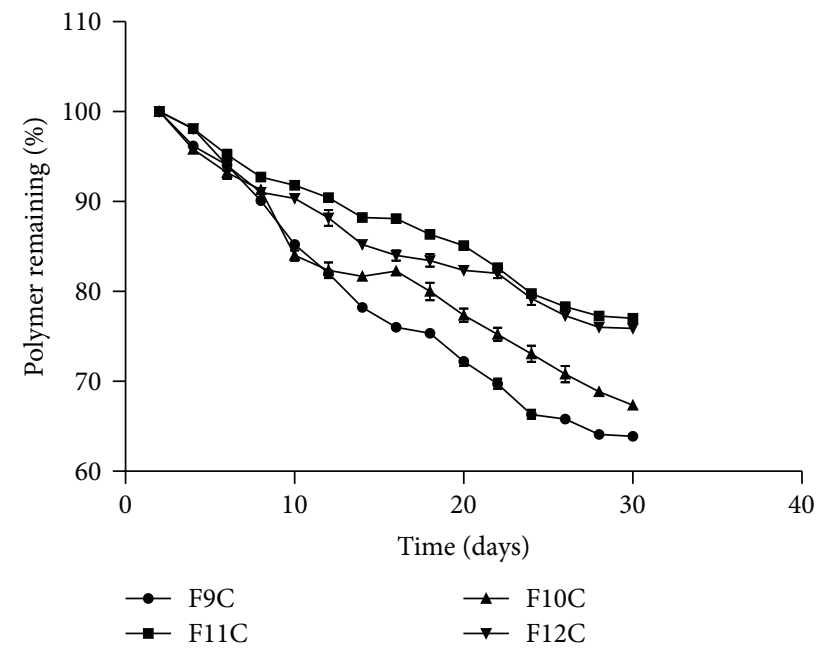

Figure 7: Polymer degradation of optimized PVA-CS hydrogel scaffold formulation.

was obtained as shown in Figure 6. The formulations with constant amount of PVA $15 \%$ and varying concentration of cross-linking agent (glutaraldehyde) from 0.5 to $2.0 \%$ were used to check the effect of cross-linking agent on drug release with respect to constant concentration of PVA. The results obtained showed that formulation F11C showed sustained release for 7 days by $72.71 \%$ while other formulations showed lesser amount of release. Increasing the PVA content causes the thicker wall formation of the matrix and causes thicker wall formation of the matrix, and so it controls the drug solution transported out of the network causing better sustained release. The rate of drug release increased with the increase in drug loading in hydrogel scaffold formulation.

3.8. In Vitro \% Polymer Remaining of Optimized PVA-CS Hydrogel Scaffold Formulation. In vitro \% polymer remaining of optimized scaffold formulation was obtained for F9CF12C formulation and found to be $63.90 \%$ (F9C), $67.34 \%$ (F10C), 77.031\% (F11C), 75.902\% (F12C) as graphically shown in Figure 7, and it was observed that optimized formulation loses nearly $23 \%$ of weight in 30 days of incubation. In

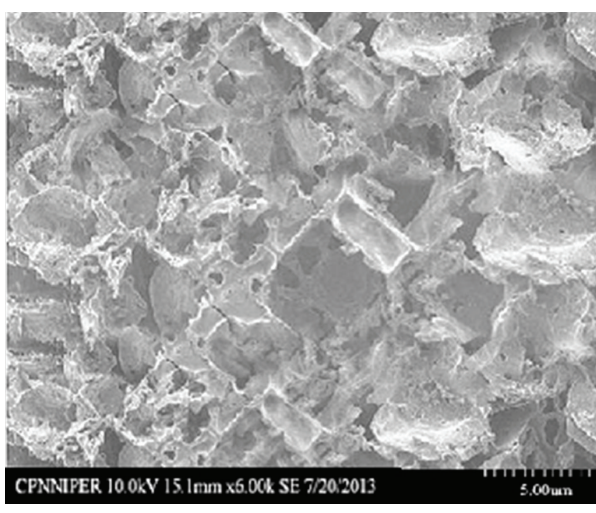

FIGURE 8: SEM Micrograph of optimized PVA-CS hydrogel scaffold formulation.

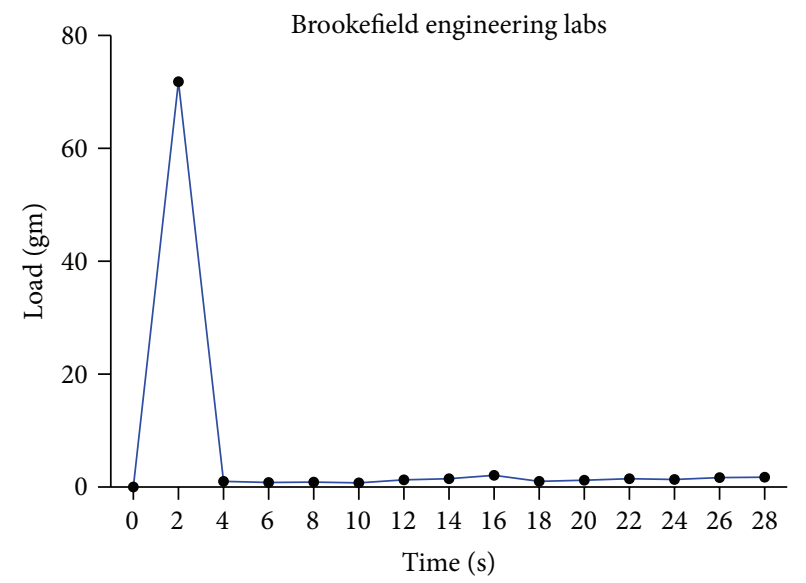

FIGURE 9: Hardness of optimized PVA-CS hydrogel scaffold formulation.

F11C formulation, \% polymer remaining was found to be maximum as compared to F9C, F10C, and F12C. Polymer degradation study is very important parameter to determine the degradation rate of scaffold formulation which is directly related to drug release pattern. Scaffold formulations retained a constant shape till the end of the degradation process. The hydrogel lose their weight due to two ways, first due to release of uncross-linked polymer (PVA) and secondly because of losing network formation which led to the leaching of polymer out of the matrices.

3.9. Morphological Studies (SEM) of Hydrogel Scaffolds. PVACS hydrogel scaffolds with well-defined internal pores and periphery were observed from SEM micrographs of hydrogels as depicted in Figure 8. The average pore size of hydrogel was found to be $5 \mu \mathrm{m}$. The ratio of PVA and glutaraldehyde influenced the swelling properties of matrices which eventually modulated their morphology and pore size. The interconnected porous structure is responsible for faster swelling of hydrogels, and these water-filled channels observed in SEM are expected to be the conduits for the transport of guest molecules during loading and release as chondroitin sulphate in cartilage repair. 


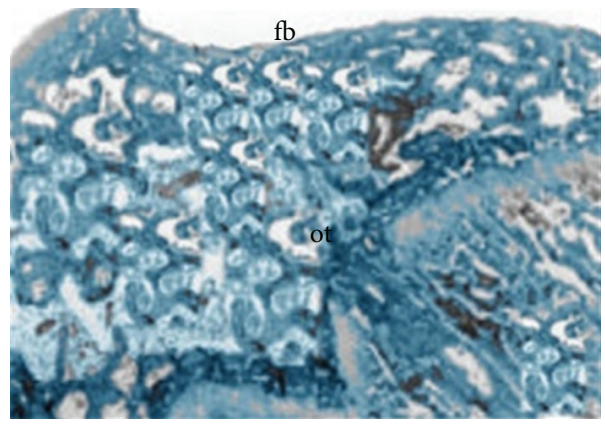

(a)

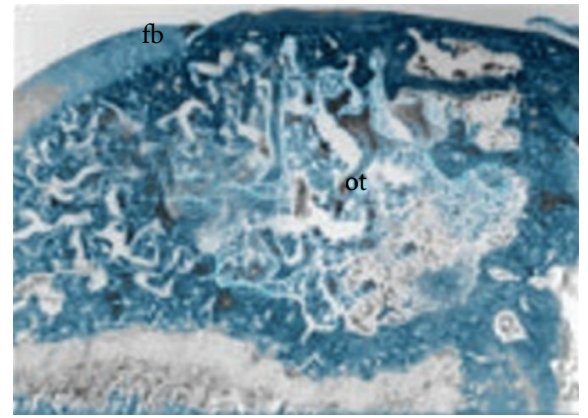

(b)

FIGURE 10: Histological photomicrographs of repaired articular surfaces stained by Masson's trichrome: (a) control group (b) implanted group after hydrogel implantation. Note the newly formed osseous tissue (ot) filling the defect and the fibrous tissue (fb) on the superficial zone. Magnification bar $=200 \mu \mathrm{m}$.

TABLE 2: Evaluation parameters of PVA hydrogel scaffold formulations (mean $\pm \mathrm{SD}, n=3$ ).

\begin{tabular}{|c|c|c|c|c|c|c|c|}
\hline \multirow{2}{*}{ F. Code } & \multicolumn{3}{|c|}{ \% Swelling index } & \multirow{2}{*}{ Viscosity $(\mathrm{Pa})$} & \multirow{2}{*}{$\begin{array}{l}\text { Apparent bulk } \\
\text { density }\left(\mathrm{g} / \mathrm{cm}^{3}\right)\end{array}$} & \multirow{2}{*}{$\%$ Water absorption } & \multirow{2}{*}{$\%$ Porosity } \\
\hline & Water & pH 6.5 & $\mathrm{pH} 7.4$ & & & & \\
\hline F1A & $1147.35 \pm 8.32$ & $1073.11 \pm 2.54$ & $1080.09 \pm 2.68$ & $3.72 \pm 0.012$ & $2.39 \pm 0.0357$ & $1248.46 \pm 7.08$ & $38.40 \pm 1.34$ \\
\hline $\mathrm{F} 2 \mathrm{~A}$ & $1030.74 \pm 8.12$ & $939.73 \pm 2.65$ & $944.22 \pm 2.92$ & $5.53 \pm 0.016$ & $2.62 \pm 0.0291$ & $1124.53 \pm 7.21$ & $34.30 \pm 1.05$ \\
\hline $\mathrm{F} 3 \mathrm{~A}$ & $908.06 \pm 7.89$ & $900.61 \pm 3.21$ & $906.05 \pm 3.21$ & $10.41 \pm 0.021$ & $2.92 \pm 0.0156$ & $997.50 \pm 6.42$ & $32.05 \pm 1.01$ \\
\hline $\mathrm{F} 4 \mathrm{~A}$ & $769.28 \pm 7.65$ & $761.33 \pm 2.75$ & $774.17 \pm 3.15$ & $17.80 \pm 0.031$ & $3.49 \pm 0.0212$ & $843.51 \pm 5.23$ & $25.60 \pm 0.92$ \\
\hline F5B & $1244.03 \pm 8.78$ & $1148.79 \pm 1.54$ & $1193.418 \pm 3.12$ & $9.11 \pm 0.013$ & $2.39 \pm 0.0347$ & $1360.51 \pm 7.51$ & $50.40 \pm 1.98$ \\
\hline F6B & $1172.48 \pm 8.56$ & $1050.64 \pm 1.75$ & $1117.53 \pm 3.78$ & & $2.86 \pm 0.0192$ & & $44.87 \pm 1.87$ \\
\hline F7B & $1148.57 \pm 8.01$ & $984.41 \pm 2.05$ & $945.79 \pm 5.09$ & $24.25 \pm 0.051$ & $2.70 \pm 0.0232$ & $1125.77 \pm 6.97$ & $39.71 \pm 1.51$ \\
\hline F8B & $900.67 \pm 7.23$ & $769.61 \pm 1.92$ & $776.43 \pm 4.20$ & $28.91 \pm 0.027$ & $3.45 \pm 0.0282$ & $992.31 \pm 5.91$ & $33.07 \pm 1.02$ \\
\hline F9C & $1108.66 \pm 7.93$ & $938.46 \pm 3.05$ & $946.04 \pm 3.91$ & $12.37 \pm 0.053$ & $2.18 \pm 0.0187$ & $1231.62 \pm 6.54$ & $42.32 \pm 1.58$ \\
\hline F10C & $1251.22 \pm 8.98$ & $1110.64 \pm 3.14$ & $1115.98 \pm 4.13$ & $17.48 \pm 0.067$ & $2.13 \pm 0.0121$ & $1399.42 \pm 7.94$ & $52.50 \pm 2.12$ \\
\hline F11C & $1589.47 \pm 9.59$ & $1230.44 \pm 3.21$ & $1235.68 \pm 6.56$ & $48.21 \pm 0.071$ & $1.82 \pm 0.0078$ & $1687.57 \pm 8.43$ & $70.30 \pm 2.86$ \\
\hline $\mathrm{F} 12 \mathrm{C}$ & $1480.20 \pm 9.52$ & $1175.85 \pm 3.01$ & $1180.51 \pm 6.02$ & $42.43 \pm 0.054$ & $1.84 \pm 0.0081$ & $1536.41 \pm 7.65$ & $66.62 \pm 2.56$ \\
\hline F13D & $1108.80 \pm 7.21$ & $1085.88 \pm 2.12$ & $1090.15 \pm 5.31$ & $27.64 \pm 0.052$ & $2.15 \pm 0.0181$ & $978.302 \pm 4.97$ & $32.05 \pm 0.92$ \\
\hline F14D & $1197.59 \pm 7.57$ & $1124.72 \pm 2.82$ & $1134.63 \pm 6.01$ & $32.42 \pm 0.034$ & $3.19 \pm 0.0312$ & $1067.29 \pm 6.12$ & $34.58 \pm 1.01$ \\
\hline F15D & $1344.83 \pm 8.91$ & $1282.44 \pm 3.15$ & $1220.428 \pm 6.25$ & $47.87 \pm 0.071$ & $1.94 \pm 0.0092$ & $1319.42 \pm 7.12$ & $61.70 \pm 2.12$ \\
\hline F16D & $1216.30 \pm 8.53$ & $1114.59 \pm 3.42$ & $1113.016 \pm 6.23$ & $39.87 \pm 0.057$ & $2.60 \pm 0.0257$ & $1443.35 \pm 7.95$ & $47.14 \pm 191$ \\
\hline
\end{tabular}

3.10. Hardness (Texture Analyzer) of Hydrogel Scaffolds. Hardness of hydrogel scaffolds depends on the viscosity of hydrogels. The hardness of optimized hydrogel scaffolds was found to be $71.8 \mathrm{gm}$ as shown in Figure 9. It was observed that the higher the viscosity of hydrogel, the higher will be the hardness. But the hardness value also may affect the swelling rate above ratio due to decrease in network volume of hydrogels which is determining factor for water preservation efficiency.

3.11. In Vivo Results. The histological results of repaired articular defects in the control and implanted groups are shown in Figures 10(a) and 10(b) after 14 days of hydrogel implantation. In the control group, the defect was not well integrated with the articular cartilage around the defect, resulting in irregular surfaces (Figure 10(a)). In implanted surfaces the hydrogel scaffold filled the defect and was well integrated to the implant site due to the growth of surrounding tissue into the hydrogel pores. The hydrogel scaffold repaired the defects, resulting in regular artificial articular surfaces (Figure 10(b)).

\section{Conclusion}

This study demonstrates the potential of chemically crosslinked PVA-CS hydrogels in regenerating cartilage tissue by evaluating in vitro and histological parameters. These hydrogel scaffolds are ideal for cartilage tissue engineering by providing suitable mechanical and physiological performance by imitating the extracellular matrix for better repair. In future, the current work can be explored by incorporating cells or signalling factors for a successful tissue engineered cartilage production to overcome the limited cartilage repaired capacity. 


\section{Abbreviations}

PVA: Polyvinyl alcohol

CS: Chondroitin sulphate

GA: Glutaraldehyde

MB: Methylene blue

PBS: Phosphate buffer saline.

\section{References}

[1] M. Cabodi, N. W. Choi, J. P. Gleghorn, C. S. D. Lee, L. J. Bonassar, and A. D. Stroock, "A microfluidic biomaterial," Journal of the American Chemical Society, vol. 127, no. 40, pp. 13788-13789, 2005.

[2] M. S. Jhon and J. D. Andrade, "Water and hydrogels," Journal of Biomedical Materials Research, vol. 7, no. 6, pp. 509-522, 1973.

[3] S. J. Bryant and K. S. Anseth, "The effects of scaffold thickness on tissue engineered cartilage in photocrosslinked poly(ethylene oxide) hydrogels," Biomaterials, vol. 22, no. 6, pp. 619-626, 2001.

[4] A. S. Hoffman, "Hydrogels for biomedical applications," Annals of the New York Academy of Sciences, vol. 944, pp. 62-73, 2001.

[5] D. A. Connell, J. Pike, G. Koulouris, N. Van Wettering, and G. Hoy, "MR imaging of thumb carpometacarpal joint ligament injuries," Journal of Hand Surgery, vol. 29, no. 1, pp. 46-54, 2004.

[6] G. Martou, K. Veltri, and A. Thoma, "Surgical treatment of osteoarthritis of the carpometacarpal joint of the thumb: a systematic review," Plastic and Reconstructive Surgery, vol. 114, no. 2, pp. 421-432, 2004.

[7] S. Huang and D. E. Ingber, "The structural and mechanical complexity of cell-growth control," Nature Cell Biology, vol. 1, no. 5, pp. E131-E138, 1999.

[8] A. J. Almarza and K. A. Athanasiou, "Design characteristics for the tissue engineering of cartilaginous tissues," Annals of Biomedical Engineering, vol. 32, no. 1, pp. 2-17, 2004.

[9] V. F. Sechriest, Y. J. Miao, C. Niyibizi, A. W. Larson, H. W. Matthew, and C. H. Evans, "GAG-augmented polysaccharide hydrogel: a novel biocompatible and biodegradable material to support chondrogenesis," Journal of Biomedical Materials Research, vol. 49, pp. 534-541, 2000.

[10] F. Allemann, S. Mizuno, K. Eid, K. E. Yates, D. Zaleske, and J. Glowacki, "Effects of hyaluronan on engineered articular cartilage extracellular matrix gene expression in 3-dimensional collagen scaffolds," Journal of Biomedical Materials Research, vol. 55, pp. 13-19, 2001.

[11] G. P. Dowthwaite, J. C. W. Edwards, and A. A. Pitsillides, "An essential role for the interaction between hyaluronan and hyaluronan binding proteins during joint development," Journal of Histochemistry and Cytochemistry, vol. 46, no. 5, pp. 641-651, 1998.

[12] K. Y. Lee and D. J. Mooney, "Hydrogels for tissue engineering," Chemical Reviews, vol. 101, no. 7, pp. 1869-1879, 2001.

[13] G. Masci, I. Husu, S. Murtas, A. Piozzi, and V. Crescenzi, "Physical hydrogels of poly(vinyl alcohol) with different syndiotacticity prepared in the presence of lactosilated chitosan derivatives," Macromolecular Bioscience, vol. 3, no. 9, pp. 455461, 2003.

[14] R. Langer and D. A. Tirrell, "Designing materials for biology and medicine," Nature, vol. 428, no. 6982, pp. 487-492, 2004.

[15] B.-S. Kim and D. J. Mooney, "Development of biocompatible synthetic extracellular matrices for tissue engineering," Trends in Biotechnology, vol. 16, no. 5, pp. 224-229, 1998.
[16] J. Varshosaz and N. Koopaie, "Cross-linked poly (vinyl alcohol) hydrogel: study of swelling and drug release behaviour," Iranian Polymer Journal (English Edition), vol. 11, no. 2, pp. 123-131, 2002.

[17] K. Pal, A. K. Banthia, and D. K. Majumdar, "Preparation and characterization of polyvinyl alcohol gelatin hydrogel: membranes for biomedical applications," Pharmaceutical Science and Technology, vol. 8, pp. 198-211, 2007.

[18] M. Jaswal and V. Koul, "Assessment of multicomponent hydrogel scaffolds of poly(acrylic acid-2-hydroxy ethyl methacrylate)/gelatin for tissue engineering applications," Journal of Biomaterials Applications, vol. 21, pp. 1-14, 2011.

[19] R. A. Gemeinhart, J. Chen, H. Park, and K. Park, "PHsensitivity of fast responsive superporous hydrogels," Journal of Biomaterials Science, Polymer Edition, vol. 11, no. 12, pp. 13711380, 2000.

[20] N. New, T. Furuike, and H. Tamura, "The mechanical and biological properties of chitosan scaffolds for tissue regeneration," Materials, vol. 2, pp. 374-398, 2009.

[21] X. Li, W. Bian, D. Li, Q. Lian, and Z. Jin, "Fabrication of porous beta-tricalcium phosphate with microchannel and customized geometry based on gel-casting and rapid prototyping," Proceedings of the Institution of Mechanical Engineers $H$, vol. 225, no. 3, pp. 315-323, 2011.

[22] A. D. Agostino, A. L. Gatta, T. Busico, M. D. Rosa, and C. Schiraldi, "Semi-interpenetrated hydrogels composed of PVA and hyaluronan orchondroitin sulphate: chemico-physical and biological characterization," Journal of Biotechnology \& Biomaterials, vol. 2, pp. 21-28, 2012.

[23] H.-W. Kim, J. C. Knowles, and H.-E. Kim, "Hydroxyapatite/poly( $\varepsilon$-caprolactone) composite coatings on hydroxyapatite porous bone scaffold for drug delivery," Biomaterials, vol. 25, no. 7-8, pp. 1279-1287, 2004.

[24] S. Zhang, "Fabrication of novel biomaterials through molecular self-assembly," Nature Biotechnology, vol. 21, no. 10, pp. 1171$1178,2003$.

[25] K. Huanbutta, K. Terada, P. Sriamornsak, and J. Nunthanid, "Advanced technologies for assessment of polymer swelling and erosion behaviors in pharmaceutical aspect," European Journal of Pharmaceutics and Biopharmaceutics, vol. 83, pp. 315-321, 2013.

[26] S. M. Malmonge, C. A. C. Zavaglia, and W. D. Belangero, "Biomechanical and histological evaluation of hydrogel implants in articular cartilage," Brazilian Journal of Medical and Biological Research, vol. 33, no. 3, pp. 307-312, 2000. 

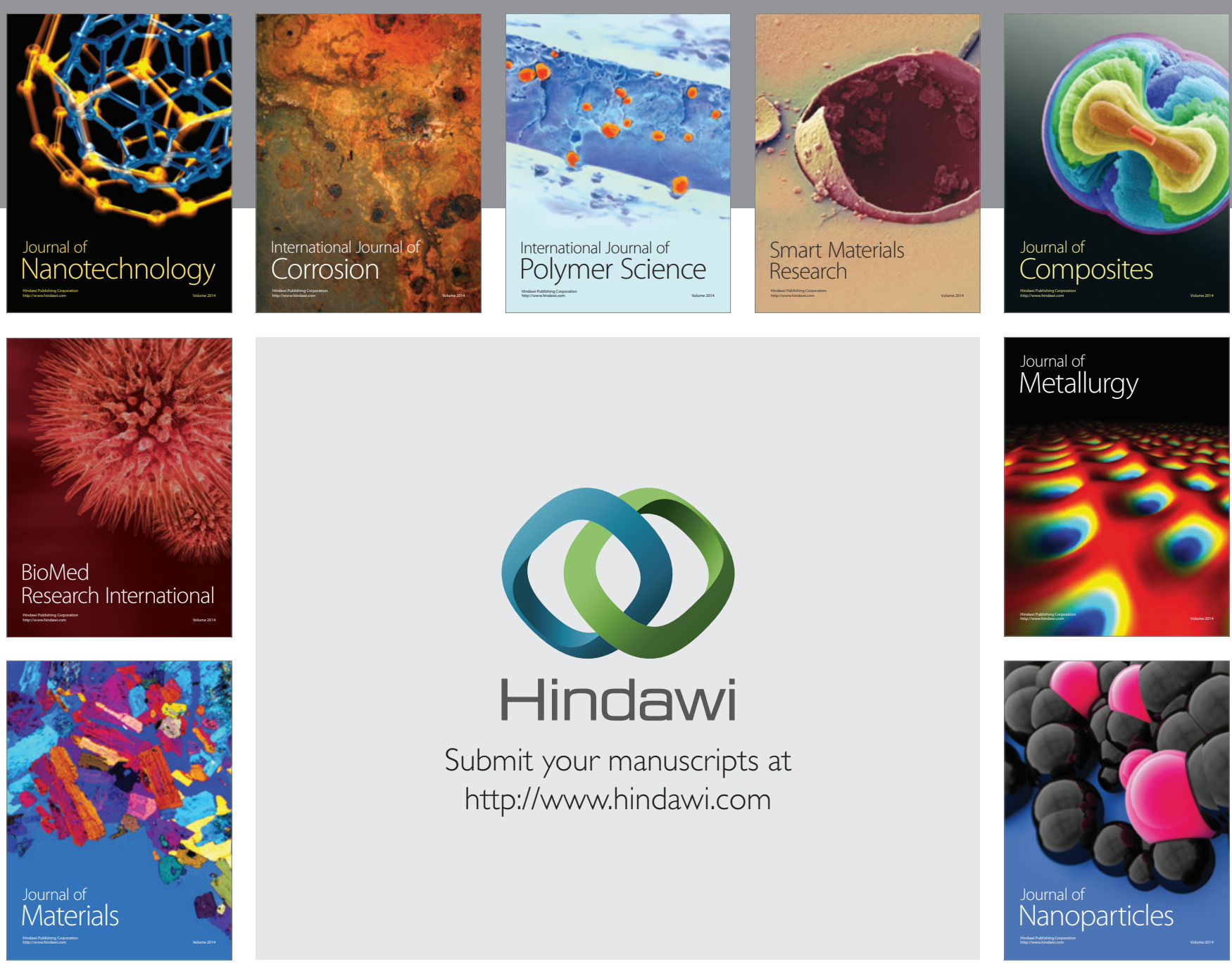

Submit your manuscripts at http://www.hindawi.com
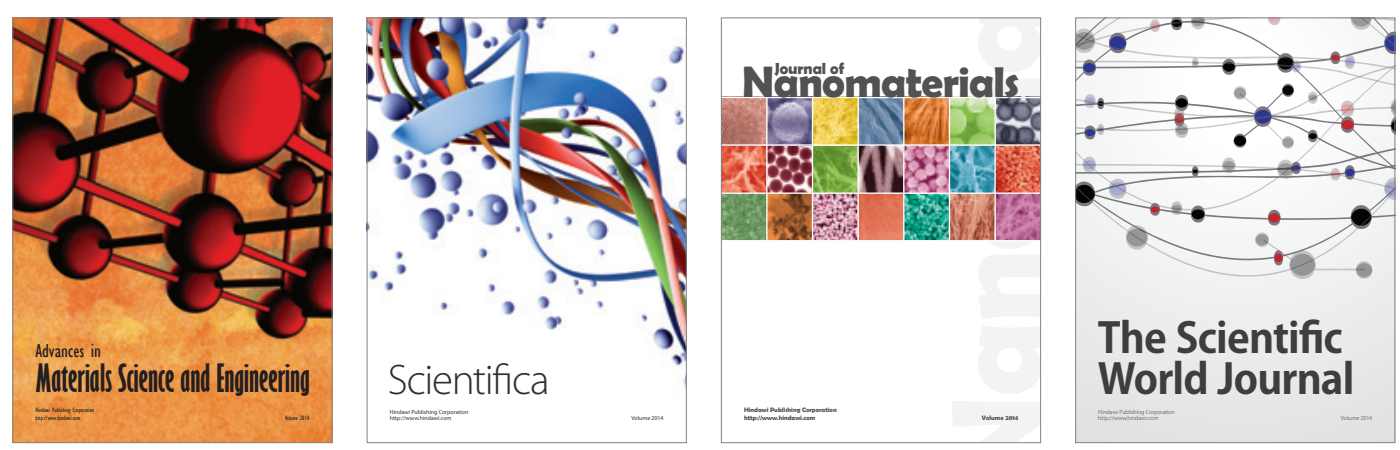

\section{The Scientific World Journal}
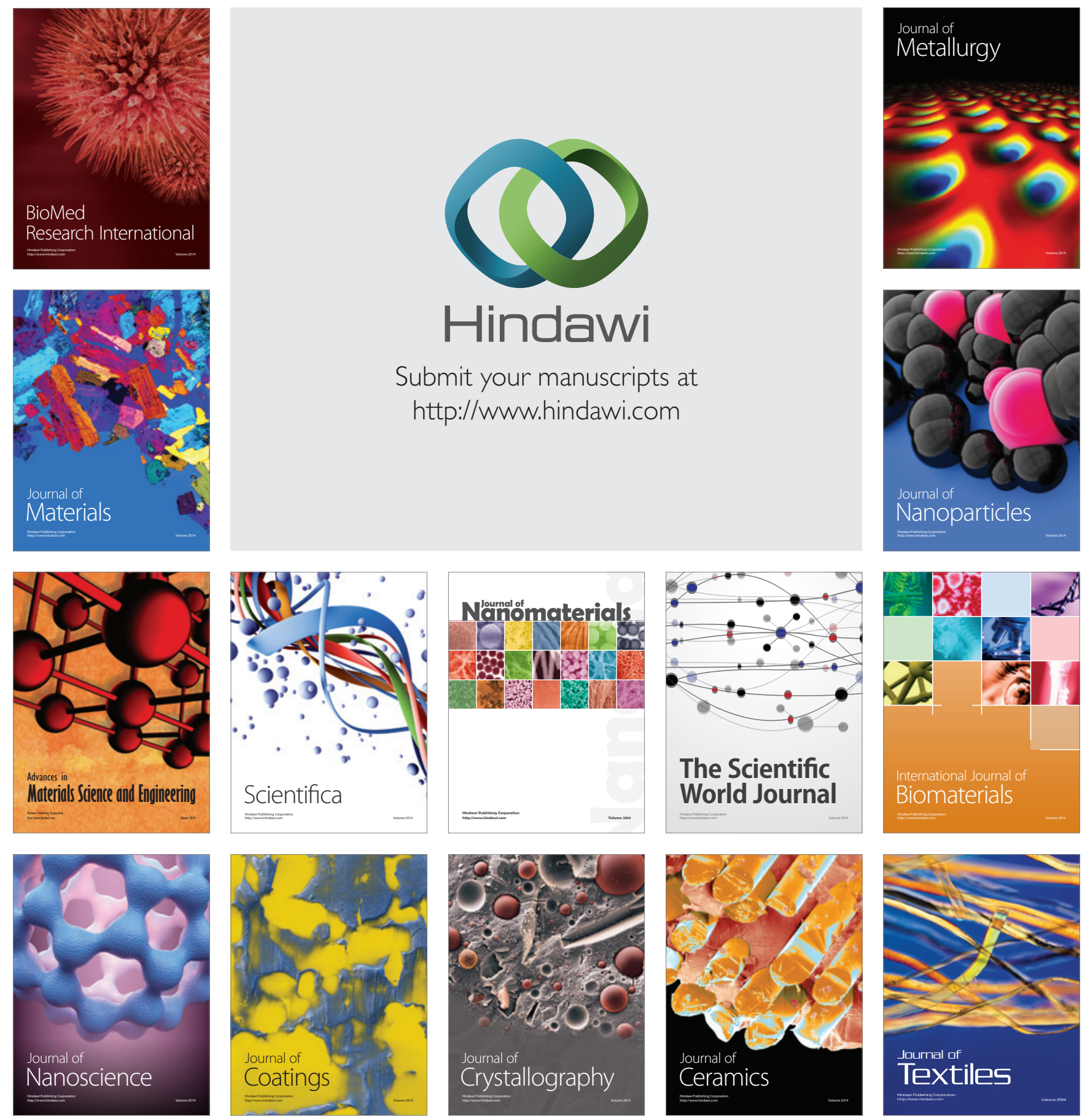